\title{
Zur Schularztfrage.
}

Von Dr. Fr. Cuntz, Schularzt in Wiesbaden.

Die Wiesbadenerschulärztlichen Einrichtungen sind durch ministerielles Rundschreiben in weiteren Kreisen zur Nachahmung empfohlen worden, und sind dieselben, mit ihrer Vorgeschichte, der genauen Dienstordnung etc. durch die Veröffentlichungen von Geh. Medicinalrath Schmid tmann (Vierteljahrsschrift für gerichtliche Medicin und offentl. Sanitätswesen 1898, 3. Folge, Bd. XVI, 1) und Stadtrath Kalle (Deutsche Vierteljahrsschrift für öffentliche Gesundheitspflege 1898, Bd.XXX, Heft II) wohl hinreichend bekannt.

Da es unseren jetzigen wie zukünftigen Schulcollegen wohl ein gewisses Interesse bieten dürfte, gerade unsere ärztlichen Anschauungen und Urtheile uber die Sache zu erfahren, so folge ich einer Anregung der Redaction, um dieselben im nachfolgenden liurz zu veröffentlichen.

Ich sehe dabei ab von dem prinzipiellen Werth und der Bedeutung der ganzen Einrichtung für Schule und Kinder -- die ja wohl keinem Zweifel mehr begegnen -, und will mich auf eine nähere Beleuchtung der drei Hauptpunkto unserer Thätigkeit beschränken. Selbstverständlich liegt es mir fern, irgendwie „lästige und überflüssige“ Kritik uben zu wollen, aber für eine definitive Beurtheilung wie weitere Ausbildung der Frage dürfte unseren Anschauungen doch eine gewisse Berechtigung zuerkannt werden müssen.

1. Die ärztliche Untersuchung der Aufnahmeclassen, d. h. der in jedem Schuljahre neu eintretenden sechsjährigen Kinder.

Fur jedes der letzteren wird ein Gesundheitsschein nach dem bekannten Muster ausgefertigt.

Was zunächst die hierzu aufgewandte Zeit betrifft, so waren für jede Classe von durchschnittlich 60 Kindern $2 \frac{1}{2}-3$ Stunden erforderlich, also $2{ }^{1 / 2}-3$ Minuten pro Kind. Dabei sind aber genauere Hör- und Sehprüfungen ausgeschlossen! Es hat sich herausgestellt, dass die Vornahme dieser Untersuchungen bei den kleinen ABC-Schützen innerhalb der ersten 3-4 Wochen des Unterrichts doch recht schwierig ist.

Die grosse Mehrzahl der Kinder ist in diesen ersten Schultagen noch so eingeschïchtert und befangen, dass auf ihre Aussagen und Antworten kein Werth zu legen ist, wenn sie überhaupt zum Antworten zu bringen sind. Auch kostet die Vornahme der Hörproben und das Erklären der Sehproben fur Analphabeten bei jedem Kinde viel Zeit, und 
es ist mir kaum begreiflich, dass Cohn (Breslau) die Sehprüfungen auch bei den Kleinen für so leicht und - so zuverlässig hält.')

Damit ist aber nicht gesagt, dass ein halbwegs aufmerksamer Beobachter nicht schon bei der ersten allgemeinen Untersuchung nennenswerth $\theta$ Grade von Schwerhörigkeit und Sehstörungen bemerken kann, wie dies auch bei unseren Untersuchungen der Fall war.

Zur Feststellung aller geringeren Mängel der Hör- und Sehschärfe bedarf es der vorläufigen Beobachtung des Lehrers und späteren gevaueren Cntersuchung durch einen Specialcollegen, mit welch' letzterer gleichzeitig die Abhülfe für das bestehende Leiden, Behandlung des Ohres, Bestimmung der erforderlichen Brillen etc. zu verbinden ist.

Wenn wir also die genauere Untersuchung von Auge und Ohr ausser Acht lassen, so dürfte die oben angegebene Zeit von $2 / 2-3$ Minuten pro Kind vollauf genügen, eine für den betreffendon Zweck ausreichende Untersuchung anzustellen und die erforderlichen kurzen Notizen in den Gesundheitsschein einzutragen. Freilich bedarf es hierzu, abgesehen von dem vorläufigen Ausfullen des Kopfes der Scheine durch den Lehrer, noch weiterer Hülfe durch eine Lehrerin, Frau des Pedellon oder zwei bis drei Mädchen der oberen Classen, die das Aus- und Ankleiden der Kinder besorgen.

Wo äussere Inspection es nicht als störend erscheinen lässt, behalten die Kinder Schube und Strüpfe, sowie Hose, bezw. Unterrock und zumeist auch Hemd an. Dabei ist die Bourtheilung der Gesammtconstitution, Beobachtung der Respiration, kurze Auscultation der Herztöne, Inspection der Mundhöhle und, bei kurzem Lufften des Hemdes, anch die des Unterleibes (Brüche), der Wirbelsäule etc. sehr wohl möglich. Eine vorgestellte, aufgehängte Landkarte oder grosse Schultafel genügt, um unberufene Zuschauer dabei fernzuhalten.

Ich darf hier hinzufïgen, dass wir auch bei den, durch die Lehrer vorzunehmenden halbjährlichen Messungen und Wägungen der Kinder von ciner Entkleidung Abstand genommen haben, aus Rücksicht auf den Zeitaufwand und die Störung des Enterrichtes. Die relativen Zahlen, die wir so erhalten, genügen vollkommen zur Beurtheilung eincr Zn- oder Abnahme.

Es ist auch bei uns die Frage aufgeworfen worden, ob die Ausstellung von diesen Gesundheitsscheinen für alle Kinder crforderlich sei and ob all' die Schreiberei und Papicransammlung mit dem wirklichen Werth im Einklang stehe.

Wenn wir das Sammeln von Material für wissenschaftliche Statistiken ausser Acht lassen und die schulärztliche Thätigkeit, wie ja auch in erster Linie beabsichtigt, auf das greifbar Praktische und Nächstliegende beschränken, so erscheinen die Gesundheitsscheine für die gesunden und normal sich entwickelnden Kinder allerdings tuberflüssig, und es dürfte genügen, solche nur für die bei der Aufnahmeuntersuchung "schwächlich und kränklicl" befundenen Kinder auszustellen, dieselben jedes Semester zu rcvidiren und mit Einträgen über die Gesammtentwicklung und den jeweiligen Zustand der beobachteten Mängel ctc. zu versehen.

Auf der andern Seite ist es aber schwer ersichtlich, wie z. B. die Subsellienfrage, die Häufung von Wirbelverkrümmungen, von Kurzsichtigkeit etc. entschieden, bezw. beurtheilt werden kann, wenn man nicht die gesammten Kinder dabei berücksichtigt; und wie will man beurtheilen, ob die Entwicklung cines Kindes während und durch die Schuljahre gestort wird, wenn man nicht einen bei der Aufnahme festgestcllten ärztlichen Befund zum Vergloiche nehmen kann?

Dass man uberhaupt zur Beurtheilung der gesammten hygienischen Verhältnisse, der Lokalitäten, wie des Unterrichts selbst, nur in dem ärztlich festzustellenden und zul controllirenden Gesundheitszustand der gesammten Kinder, den allein richtigen Maassstab zu suchen hat, ist doch wohl klar. Mit theoretischen Dednctionen, gekünstelten Ermudungsproben erreicht man nichts.

Wir haben deshalb an der Untersuchung und Ausstellung von Scheinen für sämmtliche Kinder bis jetzt festgehalten, sind aber wohl zu der Ucberzeugnng gelangt, dass die jetzige Art der Weiterfuhrung dieser Scheine nur dann einen entsprechenden Nutzen hat, wenn alle Kinder wäbrend der acht Schuljahre mehrmals einer Gesammtrevision, bezw. Untersuchung unterworfen werden, mit Befundangabe in den Scheinen. Etwa noch im dritten, fünften und letzten Schuljabr. Es wäre dies allcrdings eine nicht unbeträchtliche Erweiterung unserer Arbeitslast, fiir die auch entsprechende Honorirung zu fordern wäre.

2. Alle 14 Tage etwa halten wir sogenante "Sprechstunde" in den Sthulen ab, über deren Ausführung in der Dienstordnung Genaueres angcgeben ist. Auch hierbei bedürfen wir wieder der Mitwirkung der Lehrer, die uns auf besondere Veränderungen der Kinder aufmerksam zu machen haben. Es ist sclbstverständlich tür uns Acrzte ganz unmoglich, cine ganze Schule von 1000-1200 Kindern so genau in der Erinncrung zu haben, dass Veränderungen einzelner einem sofort auffallen; dazu wäre

1) Ich bin erst in letzter Zeit in den Besitz der neuesten Cohnschen Täfelchen und der dazu erforderlichen "Gabel" gelangt, und soll es uns freuen, wenn wir bei den nächstjährigen Untersuchungen damit rascher und leichter zum Ziele kommen. auch ein mehr als zwei- bis dreimaliger Besuch jeder Classe pro Semester nicht ausreichend.

Den grössten Werth legen wir auf den Besuch der Classen während des Unterrichts; und können wir unseren Collegen in anderen Städten nur dringend empfehlen fest darauf zu bestehen.

Einerseits lässt sich hierbei sofort dem Lehrer von Fall zu Fall klar machen, was sich für unsere Thätigkeit eignet und was nicht. Anfangs wird eben alles zur Sprechstunde geschickt, was auf eine Frage des Lehrers irgend etwas zu klagen hat.

Andererseits lassen sich nur bei Besuchen der Classen während des Lnterrichts, dessen kurz dauernde Störung nach dem Urtheil der hiesigen Schulmänner durchaus nicht von Belang ist, die Ventilation, Heizung, Haltung der Schulkinder etc. beobachten.

Dass im übrigen unsere Thätigkeit in diesen Sprechstunden wenig „ärztliches“ an sich hat, liegt auf der Hand, wenn man bedenkt, dass ja jede Verordnung, Behandlung, therapeutische Eingriffe etc., abgesehen von dringenden Fällen, vermieden werden sollen.

Neben den Fällen, in denen wir dem Lehrer, im Interesse des Unterrichts und der Schule, Aufklärung zu geben haben über Art und Bedeutung einer Erkrankung, deren Uebertragbarkeit, über erforderliche Schonung beim Schreiben, Lesen, über Dispens vom Turnen, Baden, uber Anweisung für das in der Schule unentgeltlich gelieferte nahrhafte warme Frühstuck etc., liegt uns ob, in geeigneten Fällen, durch gedruckte Mittheilungen (siche Formular) die Eltern auf eino bestehende Erkrankung ihrer Kinder und eine eventuell erforderliche Behandlung, Operation etc. aufmerksam zu machen.

Diese „Mittheilungen“, die erforderlichen Falles auch bei der Aufnahmeuntersuchung schon ausgefüllt und versandt wcrden, haben, vom rein ärztlichen Standpunkt aus, einen meist nur zweifelhaften Erfolg gehabt.

Abgesehen von den Extremen, wo die betreffenden Eltern personlich durch den Lehrer für die gewordene Aufklärung sich bedankt und diesclbe sofort befolgt haben und wo dieselbe in beleidigender Form zurückgewiesen wurde, war es bei der Mehrzahl fraglich, ob es uberhaupt als Erfolg anzusehen ist, wenn die Kinder auf die Mittheilung hin zwar bei ihrem Arzte waren, angeblich eine Behandlung angeordnet, eine Operation für die „nächsten Ferien“ in Aussicht genommen ist, aber mit der Erkrankung bleibt es beim Alten!

Es ist dies ein Punkt, wo eine Collision mit den Hausärzten sehr leicht moglich ist. Besteht eine Meinungsverschiedenhcit uber Nothwendigkeit und Nutzlichkeit einer Behandlung oder Operation, so hat es der Hausarzt sehr leicht, den Schularzt zu discreditiren, durch absichtliche oder unabsichtliche Bemerkungen, wie „das ist ja überflussig, ich kenne das Kind ja doch besser, das wird von selbst wieder gut" etc. etc.; schickt aber der Schularzt nach einiger Zeit erneut eine "Mittheilung" an die Eltern, so erblickt der Hausarzt hierin leicht eine Kritik seines Verhaltens, eventuell auch sciner Behandlungsweise.

Es muss deshalb vom Schularzt erwartet werden, dass er bei langdauernden, schwierig zu behandelnden Erkrankungen nicht unnöthiger Weise die Eltern belästigt und beunruhigt und den Hausarzt "reizt"!

Chronische Mittelohreiterungen, alte Blepharadenitis, Prurigo, Psoriasis vulgaris etc. bedürfen Zeit und Ausdauer, wenn sich die Eltern des Patienten, bei der nur allzu häufigen Renitenz und Indolenz, uberhaupt entschliessen etwas dagegen thun zu lassen. Ueberflussig sind auch Mittheilungen über harmlosen Naevus, stabile rachitische Verkrümmungen (wenn nicht sehr hochgradig), in Rückbildung begriffene Cornealflecken, oder gar ein unschuldiges Flohstichexanthem!

Abgesehen von dem Interesse für die Schule, das doch auch bei den Hansärzten wie bei jedem Gebildeten rorausgesetzt werden muss, förderı die Schılärzte ja auch geradezı das geschäftliche Interesse der ersteren, durch Drängen auf ärztlichc Behandlung, Zuweisung von sonst vielleicht nicht beachteten Erkrankungen ete., also Grund genug für gegenseitiges Entgegenkommen. Zu einem nennenswerthen Conflict zwischen Schulund Hausarzt ist es bei uns noch nicht gekommen, und kann ein solcher bei nur halbwegs guten collegialen Verhältnissen wohl uberall vermieden werden.

3. Die hygienische Eeberwachung der Schullokalitäten etc. hat uns bis jetzt nicht oft Vcranlassung zur Thatigkeit gegeben. Obgleich die letztere hierbei grosscntheils "polizeilicher" Natur ist, so ist es doch richtiger, wenn der Arzt sich um diese Sachen kummert, anstatt alles dem Lehrer zu uberlassen. Auch werden durch unsere zeitweise ausgeübte Controlle die Lehrer gewiss veranlasst, auch ihrerseits genauer auf Heizung, Beleuchtung, Ventilation etc. zu achten.

Es bestehen für alle diese Fragen ja schon die sehr eingehehenden jährlichen Revisionen des Medicinalbeamten mit den bis ins kleinste gehcnden Fragebogen, und können deshalb unsere Ausstellungen und Beobachtungen nur uutergeordneter Natur sein, meist wohl auch Wiederholungen der schon vom Kreisphysikus gerügten Missstände.

lmmerhin haben unsere Beschiverden, als Grındlage für die geschäftsmässigen Meldungen der betr. Rectoren dazu gedicut, die städtischen Baubehörden zu raschercm Vorgchen und definitiver Beseitigung der Missstände zı veranlassen.

Die Physikatsberichte uber Schulrevisionen werden zweifellos längere 
Zeit brauchen, bis sie den vorgeschriebenen [nstanzenweg durchgelaufen und an die richtige Adresse gelangt sind, wenn sie unterwegs nicht vollkommen im Aktenschrank verschwinden.

Um nur einige Punkte hervorzuheben, wo hierbei unsere Thätigkeit mit Erfolg eingegriffen hat, so seien erwähnt: ungenügende Gasbeleuchtung in einigen Klassenzimmern, Verbot eines Zimmers ohne jegliche Ventilationsvorrichtung für Unterrichtszwecke, mangelhafte Belichtung von Wandtafeln, Klassenüberfüllung, Ueberanstrengung einer Aufnahmeklasse durch ungeeignete Stundenvertheilung, kleinere Missstände auf Aborten, Entfernung eines Trinkhahnes vom Abortgebäude etc. etc.

Je mehr freilich die alten Schulgebäude verschwinden und neueren, auch in hygienischer Hinsicht besseren Schulpalästen Platz machen, desto weniger wird es da für uns Schulärzte zu thun geben. Immerhin dürften die Collegen in anderen Städten in dieser Hinsicht noch Arbeit genug finden, mehr als wir hier in Wiesbaden.

Ferner sind bis jetzt hier, vermuthlich auch anderswo, die Pläne fur neu zu errichtende Schulbauten niemals einem ärztlichen Begutachter vorgelegt worden; selbst der königl. Kreisphysikus hat nur das fertige Gebäude zu beurtheilen!1) Auf unser Betreiben werden von nun an alle Pläne für städtische Schulbauten auch den Schulärzten zur Aeusserung vorgelegt, ehe sie zur Ausführung genehmigt werden. Freilich müssen wir uns hierbei, bis wir mehr in diesen Fragen bewandert sind und mehr Erfahrungen gesammelt haben, auf eine „bescheidene“ Kritik beschränken; für Innehaltung der gesetzlichen Vorschriften ist die Baubehörde ja verantwortlich.

$\mathrm{Zu}$ unserer Thätigkeit gehören nun ausser den oben erwähnten drei Hauptpunkten noch manche andere, die sehr wohl nähere Besprechung und Auseinandersetzung, ja selbst Kritik erfordern, ich muss aber vorläufig hierauf verzichten; wir sind selbst über dieselben noch nicht zu einem abschliessenden Urtheile gelangt und ändern und versuchen noch manches.

So vor allem die "Vorträge in den Lehrerversammlungen“, gegen die wir, als regelmässige Verpflichtung, nach den gemachten Erfahrungen, Bedenken verschiedener Art haben: ferner die controllire nden Besuche im Hause eines erkrankten Schulers, bei denen jeder Zwang gesetzlich unzulässig ist, die aber für die Schulbehörde von grossem Werth sind; die wirksame Handhabung der Anzeigepflicht bei Infectionskrankheiten, beziehungsweise die geeignete Benachrichtigung der Schule besonders uber Wiedergenesung, geschehene Desintection etc.; die erfolgreiche Bekämpfung der Läuseplage durch eventuell zu ergreifende $Z_{\text {wangsmaassregeln etc. etc. }}$

Hierzu bedarf es einer gellauen Kenntniss und Anlehnung an die gesetzlichen Bestimmungen, und ist deshalb die Mitwirkung des hiesigen Regierungsmedicinalraths und des Königlichen Kreisphysikus bei unseren Berathungen von grosser Wichtigkeit.

Erwähnen möchte ich noch, dass dahier, mit Genehmigung des Herrn Ministers, jeweils einem der Schulärzte Sitz und Stimme in der städtischen Schuldeputation zugestanden ist, und ich möchte die Collegen bitten, auch in anderen Städten dieselbe Befugniss für sich erstreben zu wollen. Trotz einer noch kurzen Erfahrung darf ich doch behaupten, dass bei den Berathungen dieser Behörde eine ganze Anzahl Fragen zur Entscheidung gekommen sind, bei denen ein schulärztliches Urtheil von grossem Werth, um nicht zu sagen ausschlaggebend war; so besonders bei der damit verbundenen Verpflichtung zur Begutachtung von Urlaubsgesuchen, Pensionirungen und Anstellungen von Lehrern.

Hoffentlich findet die ganze Schularztfrage anderwärts die gleiche Bereitwilligkeit und das gleiche Verständniss bei den städtischen Körperschaften wie hier, und darf ich dabei des thatkräftigen Eintretens des Stadtraths Kalle, des eigentlichen Vaters der hiesigen Einrichtung, wie auch des Entgegenkommens und der bereitwilligen Mitarbeit der Schulbehorde und hiesigen Lehrerschaft nicht vergessen.

Wenn ich zum Schluss unser Urtheil uber unsere "schulärztliche Thätigkeit" zusammenfasse, und ich weiss mich hierbei im Einklange mit der überwiegenden Mehrzahl meiner hiesigen Collegen, so lautet dies, in Uebereinstimmung mit den Ausführungen des Geh. Med.Raths Schmidtmann: "Soweit die kurze Zeit des Bestehens ein abschliessendes Urtheil ermöglicht, hat sich nicht nur die Durchfuhrbarkeit, sondern auch der Nutzen und Werth der Anstellung von Schulärzten durchaus bewiesen, wenn auch in einzelnen Punkten die bisherigen Erfolge den Erwartungen nicht in wünschenswerthem Masse entsprochen haben, und noch manche "Unebenheiten und Steine des Anstosses" zu beseitigen sind." 